\title{
BMJ Open Monitoring young lifestyles (MyLife) - a prospective longitudinal quantitative and qualitative study of youth development and substance use in Norway
}

Geir Scott Brunborg, Janne Scheffels, Rikke Tokle, Kristin Buvik, Elisabeth Kvaavik, Jasmina Burdzovic Andreas

To cite: Brunborg GS,

Scheffels J, Tokle R, et al. Monitoring young lifestyles (MyLife) - a prospective longitudinal quantitative and qualitative study of youth development and substance use in Norway. BMJ Open 2019;9:e031084. doi:10.1136/ bmjopen-2019-031084

- Prepublication history for this paper is available online. To view these files, please visit the journal online (http://dx.doi. org/10.1136/bmjopen-2019031084).

Received 15 April 2019 Revised 29 August 2019 Accepted 01 October 2019

D) Check for updates

(c) Author(s) (or their employer(s)) 2019. Re-use permitted under CC BY-NC. No commercial re-use. See rights and permissions. Published by BMJ.

Department of Alcohol, Tobacco, and Drugs, Norwegian Institute of Public Health, Oslo, Norway

Correspondence to

Dr Jasmina Burdzovic Andreas; jabu@fhi.no

\section{ABSTRACT}

Purpose The Monitoring Young Lifestyles (MyLife) project was initiated as an integrated quantitative and qualitative prospective investigation of correlates, causes, and consequences of adolescent substance use and other addictive behaviours in Norway.

Participants The MyLife cohort was recruited from middle schools in Norway, which were selected from low, medium and high standard of living areas in both rural and urban regions of the country. A total of 3512 eighth, ninth and tenth graders ( $55 \%$ girls) from 33 schools were enrolled in the quantitative project arm (QT), while a total of 120 eighth graders ( $52 \%$ girls) from six schools were enrolled in the qualitative project arm (QL).

Findings to date QT baseline was conducted in the fall of 2017, when 2975 adolescents completed an online questionnaire at school during a regular class time. A total of 2857 adolescents participated in the first QT follow-up 1 year later. QL baseline was conducted across the fall semesters of 2014 (one class) and 2015 (five classes), when a total of 118 eighth graders completed face-to-face interviews. QL follow-ups were conducted in the spring of 2015 and fall of 2017 ( $n=98)$ for group interviews, and in the spring of 2017 and 2018 ( $n=95)$ for individual interviews. In terms of additional data sources, a total of 3035 parents consented to own participation, of which 1899 completed a brief online questionnaire at QT baseline in late 2017. School principals completed brief surveys at the same time.

Future plans Both QT and QL arms have planned followups through 2021. Consents were obtained for individuallevel linkages of adolescent and parental quantitative surveys to each other, as well as to the information available in multiple national registries and databases. These supplemental data sources will provide key information on additional putative exposures as well as on the long-term health, educational, and social outcomes of the MyLife participants.

\section{INTRODUCTION}

Adolescent substance use remains a significant public health concern, as early substance use is associated with a range of adverse

\section{Strengths and limitations of this study}

- To the best of our knowledge, Monitoring Young Lifestyles (MyLife) is the first integrated quantitative and qualitative prospective study of youth in Norway that specifically focuses on substance use and related behaviours.

- MyLife quantitative arm encompasses adolescent participants of wider age ranges (three grades/ cohorts, 13 to 16 years old at baseline), sizeable samples (approximately 1000 per grade/cohort), and multiple and balanced follow-ups (annual, for 5 years).

- MyLife qualitative arm encompasses sizeable samples (approximately 120 eighth graders from six schools/classes) and multiple follow-ups (a total of five group and individual interviews over the period of 6 years).

- The consent and recruitment procedures were limited by privacy and confidentiality concerns, with possible implications for representativeness and related inferences.

- Future plans involve individual-level linkages of adolescent and parental quantitative surveys to each other, as well as to the individual-level health and social registries data to ultimately extend the current knowledge on adolescent development in general, and on substance use and addictive behaviours in particular.

psychological, social and health outcomes. ${ }^{1-3}$ In addition, the Global Burden of Disease project ranks substance use and the related disorders among the most important risk factors for mortality and disability in high-income countries such as Norway. ${ }^{4}$ This burden is especially salient among the youngest, as mental health and substance use disorders are the primary contributors to disability in youth from high-income countries. ${ }^{6}$ For example, among those between 5 
and 14 years of age in Norway, alcohol use remains the number one behavioural risk for mortality and number three risk for disability. ${ }^{5}$

Understanding the use of alcohol, tobacco and other drugs, as well as the causes and consequences of these behaviours as they occur early in life is therefore of key public health importance, both in Norway and internationally. The relevant research no longer seeks to simply identify the most important risk and protective factors ${ }^{7-9}$; instead, it increasingly aims to understand their complex contribution to varied patterns and developmental trajectories of early drinking, smoking and drug use. ${ }^{10-17}$ Indeed, understanding the developmental course of substance use has become central to understanding the causes, onset, timing, duration and consequences of these behaviours. More importantly, understanding how different substance use patterns develop and progress over time and across various subpopulations ultimately informs not only our understanding of the associated risk and protective factors, but also of the potential prevention and treatment strategies. ${ }^{7818}$

Such questions are best explored within the developmental psychopathology and epidemiology frameworks and social-ecological theories of human development. ${ }^{19-27}$ These approaches study individuals within their social contexts (ie, families, schools, cultures, etc.) and focus on causal mechanisms underlying developmental shifts towards or away from pathological outcomes and problem behaviours such as substance use. ${ }^{20-22} 27-30$ While these fields have been traditionally dominated by quantitative approaches and statistical analyses, integration of qualitative methods into core developmental psychopathology frameworks can offer unique advantages. ${ }^{31} 32$ Specifically, qualitative methods address the 'why' questions and provide insights into the larger socio-cultural contexts in which individuals develop ${ }^{33}{ }^{34}$.Thus, the results from the integrated studies offer both depth and breadth in understanding of youth development and youth cultures. For example, repeated interviews with adolescents can help us map and describe the complex social and cultural processes underlying their use of alcohol, tobacco or drugs. Yet, multidisciplinary and mixed-methods studies of early substance use remain few and far between, constrained by the high-risk samples, cross-sectional designs and general non-reliance on developmental framework. 3536

These research challenges and the resulting knowledge gaps are especially evident in Norway, where substance use remains a significant public health issue and policy priority. ${ }^{37}$ While numerous Norwegian cross-sectional reports examined various aspects of early substance use, ${ }^{38-46}$ such studies could not fully address the key questions of causal pathways and intraindividual developments over time. A handful of notable longitudinal reports also remain somewhat limited: even though they are based on large, well-established prospective cohorts (eg, the Young in Norway Longitudinal Study ${ }^{47}$ or the Tracking Opportunities and Problems Study ${ }^{48}$ ) these projects were not established with the specific purpose of investigating early substance use. Finally, a comprehensive qualitative longitudinal investigation of substance use among youth, the surrounding cultures and underlying processes has not been undertaken in Norway so far.

Given the above-identified gaps, the Monitoring Young Lifestyles (MyLife) project was initiated as a largescale multidisciplinary and mixed-methods prospective investigation of early substance use and other addictive behaviours, their normative and non-normative developmental courses, their varied causes and consequences, putative comorbidities and underlying psychological and social processes and mechanisms. Our primary research questions will therefore focus on identification and examination of risk and protective factors associated with substance use patterns across adolescence. Closer examination of sensitive developmental periods and larger contextual factors in relation to changes both across and within individuals will also be prioritised. These questions will be examined both quantitatively (ie, examination of adolescents' repeated surveys, parental surveys and various administrative data sources) and qualitatively (ie, examination of adolescents' repeated individual and group-based interviews using thematic content and narrative analyses). Further, we aim to both quantitatively and qualitatively explore numerous understudied and emerging questions, including the problematic use of social media and video games, ${ }^{49-52}$ the role of alcohol and drug use opportunities, ${ }^{39} 4253$ putative gender, ethnic and socioeconomic variations and differences, ${ }^{15} 5455$ resilient outcomes in face of multiple risk factors ${ }^{41}$ and shifting cultural norms and behaviours surrounding early substance use. ${ }^{455-59}$ Finally, a wider range of research questions and outcomes extending beyond adolescence can eventually be addressed through various secondary projects and the planned individual-level linkages of quantitative data with other administrative and health data sources readily available in Norway. ${ }^{60}$ The study ultimately aims to narrow the existing research gap, to inform relevant public health policies, and to improve prevention and intervention strategies concerning early substance use and other addictive behaviours. This report describes the MyLife project, its design and sampling, recruitment and data collection for quantitative and qualitative project arms, core cohorts and selected preliminary results.

\section{COHORT DESCRIPTION}

\section{MyLife core project design and procedures}

A prospective longitudinal design was selected as the most scientifically and logistically feasible one, and was fully integrated into the MyLife quantitative and qualitative project arms (described below). Middle school students were identified as the most appropriate target population, given that they were (a) of ages immediately preceding the largely normative onset of studied behaviours and (b) cognitively capable of informed assent and independent 


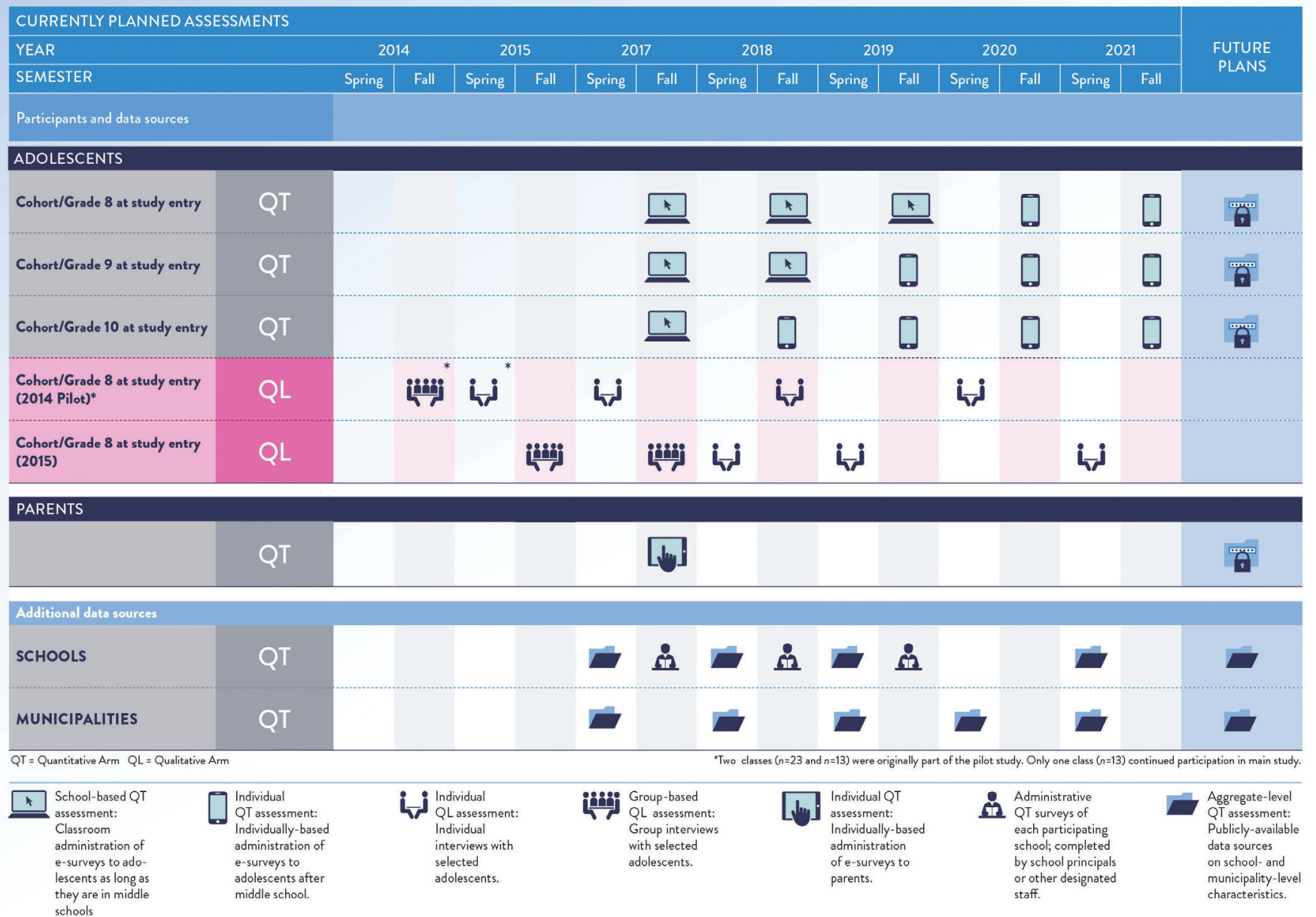

Figure 1 MyLife study design, data sources and planned assessments.

survey and interview participation. Consequently, middle schools were identified as the most appropriate recruitment and initial assessment platforms. The project consists of three main components, described below and in figure 1 :

\section{Quantitative arm(QT)}

This arm was designed as a multi-cohort study with five annual prospective assessments (aka, 'accelerated longitudinal design' (ALD)). ALD was selected as the most efficient design, given the budgetary, time and staff constraints. ${ }^{61-63}$ Additional features include integration of multiple cohorts (three school cohorts; middle school grades 8, 9 and 10 at QT baseline), sizeable samples (approximately 1000 students from each cohort/grade) and relatively frequent and balanced follow-ups (annual, for 5 years). Data collection windows for all five annual assessments were set during the Fall semester, with closing at the last day of that calendar year (ie, September through $31^{\text {st }}$ December). The chosen QT design fully reflects our key theoretical models and scientific aims while optimising data collection time and robustness to dropout. ${ }^{6164}$ It also facilitates modelling of complex trajectories, patterns and groups; of conjoint and comorbid outcomes over time; and of critical developmental events, periods and shifts. ${ }^{63-67}$
Qualitative arm (QL)

This arm was designed as a semi-structured longitudinal study encompassing five semi-structured group- and individual interview assessments over the period of 6 years. Enrolment and interviews schedule (ie, timeline and balancing between the individual and group interviews) were based on methodological and structural considerations ranging from availability of research funds and staff, to availability of participating schools and students. This arm featured a single-cohort design, where only eighth graders were included at baseline. The developmental approach was chosen in order to understand a broad range of adolescents' experiences over time, including alcohol, tobacco and drug use. ${ }^{68}$

\section{Parental QT reports and additional data sources}

Parental reports completed at QT baseline provide additional information beyond the youth self-reports, whereas the school principals' reports completed at QT baseline provide information on school characteristics of relevance to both quantitative and qualitative contextual analyses. Further, publically accessible administrative data on schools and communities will also facilitate quantitative and qualitative examination of larger structural factors potentially shaping developmental trajectories and health outcomes of interest. Finally, adolescent and 
parental QT responses can be linked to a range of objective individual-level indicators of health, adjustment and well-being available through various national registries. ${ }^{60}$ Study design, all participants, data sources, and planned timelines are shown in figure 1.

It should be noted that the original MyLife design proposed overlapping QL and QT arms and samples, as well as the common baseline for the Fall semester of 2015. The separation of data collection schedules for the two arms resulted from a lengthy evaluation process by the Norwegian Data Protection Authority. Nevertheless, the MyLife project still facilitates mixed-methods analyses, although not at the individual student level. ${ }^{3459}$

\section{Setting}

The setting for the study is Norway, a high-income sparsely-populated country in Northern Europe characterised by a generous welfare state with public education and universal healthcare systems. Norway is ranked number one on the Inequality-adjusted Human Development Index. ${ }^{69}$ The main behavioural risk factors for both years of life lost and years lived with disability in the population between 15 and 49 years of age are tobacco, alcohol, and drug use. ${ }^{5}$

\section{Sampling and recruitment}

The first step in sampling procedures involved selection of geographical areas. To ensure geographical and sample diversity, 5 out of 19 counties in Norway were chosen for study inclusion: one each from the north (Troms), from the middle (Sør-Trøndelag), from the west (Møre og Romsdal), from the south (Rogaland), and from the east (Buskerud) of the country, see figure 2. The second step involved ensuring representation of both urban and rural areas. Within each county, we first selected the schools from the largest city in the county, and then schools from rural municipalities - but for practical reasons, still within a 2 hours drive from the largest city. Schools with fewer than 50 students were excluded because of a poor cost-benefit ratio. The third step involved ensuring representation of low, middle and high standard of living communities. To this end, we used the Standard of Living Index (SLI) - a standardised indicator available from Statistics Norway for all Norwegian municipalities up to 2008 and reflecting community-level characteristics ranging from social security, single parent and disability payments, to mortality and unemployment rates. ${ }^{70}$ The municipalities within counties, and the districts within cities were sorted into low, middle and high SLI categories using tertile splits. Municipalities/districts were

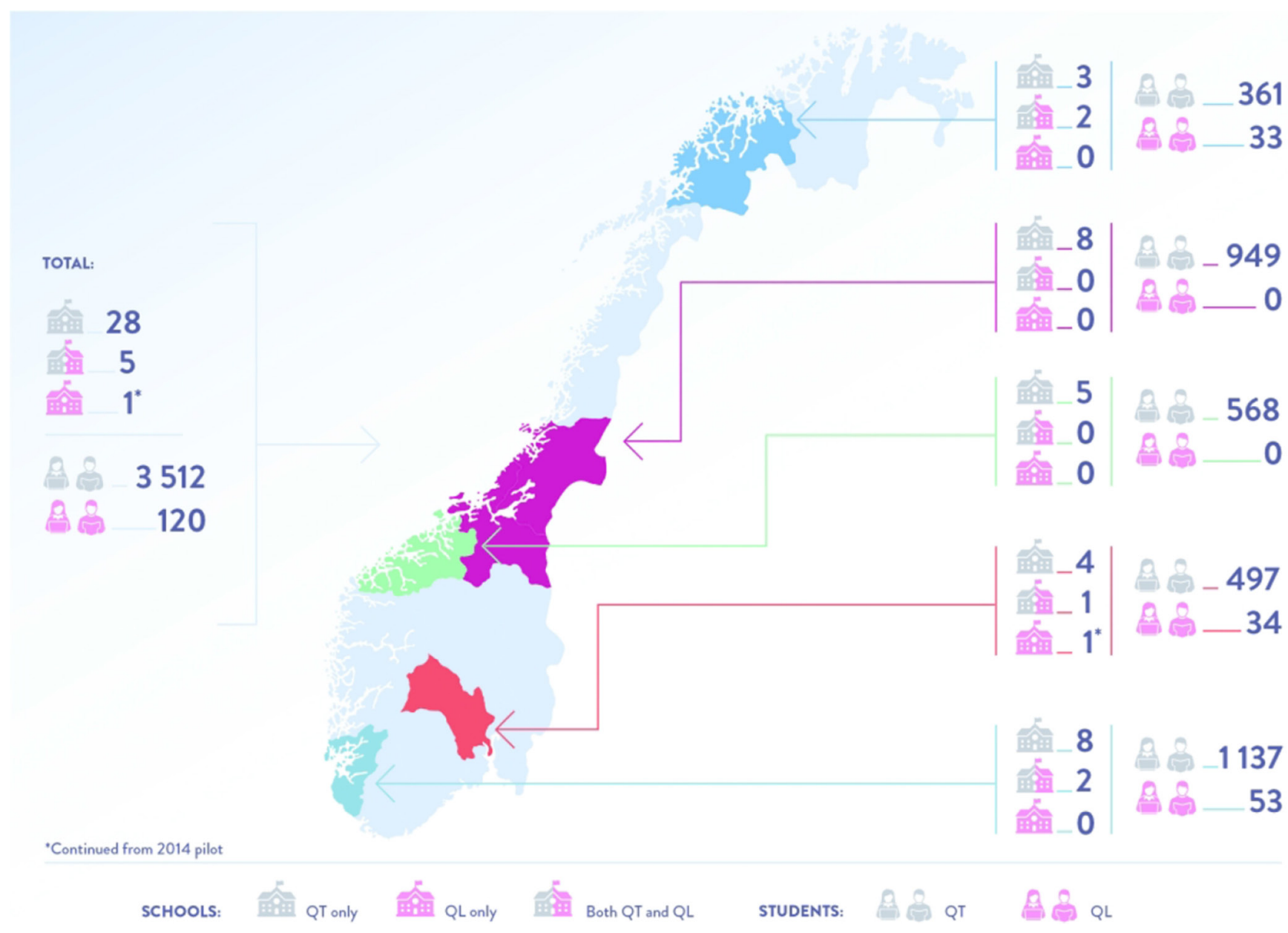

Figure 2 MyLife cohort geographical representation. 
drawn from the sorted list using a random number generator so that $30 \%, 40 \%$ and $30 \%$ of the target sample would be from low, medium, and high SLI communities, respectively.

In almost all cases, there was only one eligible school within each geographical area. In cases with more than one school, the target school was selected through a random number generator. This procedure resulted in 42 schools with upwards of 9500 middle school students (see figure 2 for details) suitable for both QT and QL arm participation. Two additional schools previously identified in the pilot project were eligible for QL.

The enrolments in the identified schools ranged from 54 to 529 middle school students. All 42 schools were contacted and invited to participate in the QT arm. Nine schools declined, leaving 33 schools with a total enrolment of about 7000 students potentially available for study inclusion. A total of seven schools were invited to participate in the QL arm; five schools identified through the procedure above were eligible for new participation, while two schools identified during the pilot study were eligible for continued participation. One school declined, leaving a total of six QL eligible schools. The ultimate aim was to enrol full eighth, ninth, and tenth grade cohorts from each school if possible (for QT) and one eighth grade class/cohort per school (for QL). These school recruitment procedures were completed in 2015 and are shown in figure 3 (for QT) and figure 4 (for QL).

\section{Informed consent}

General procedures

Because of the respondents' young age, informed parental consent was required before the children could be invited to participate, or give assent for own participation in the MyLife study. The schools were provided with information packages containing a printed booklet describing the project in plain language, explicit consent form and a secure return envelope; this package was administered to all students during regular class time. Students were asked to take the package home, share it with their parents and to return sealed envelopes with completed consent forms to their teachers by a deadline. In order to estimate as accurate as possible response and consent rates, we asked that the forms be returned even if no consent for study participation was granted. Those with parental consent were asked to assent for own participation immediately preceding the QT and QL baseline assessments.

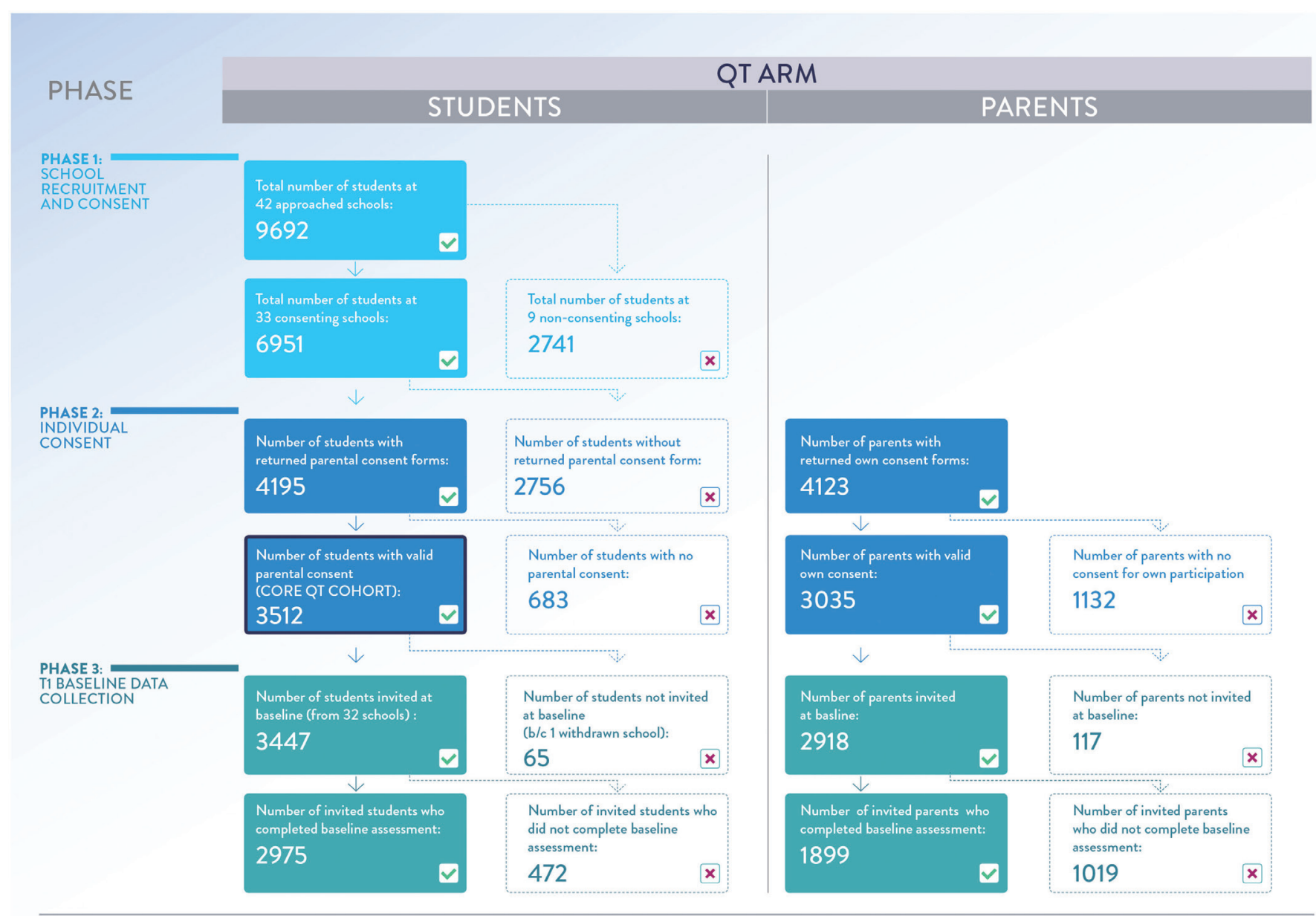

Figure 3 Flow chart for the recruitment of the quantitative arm (QT) of the MyLife study. 


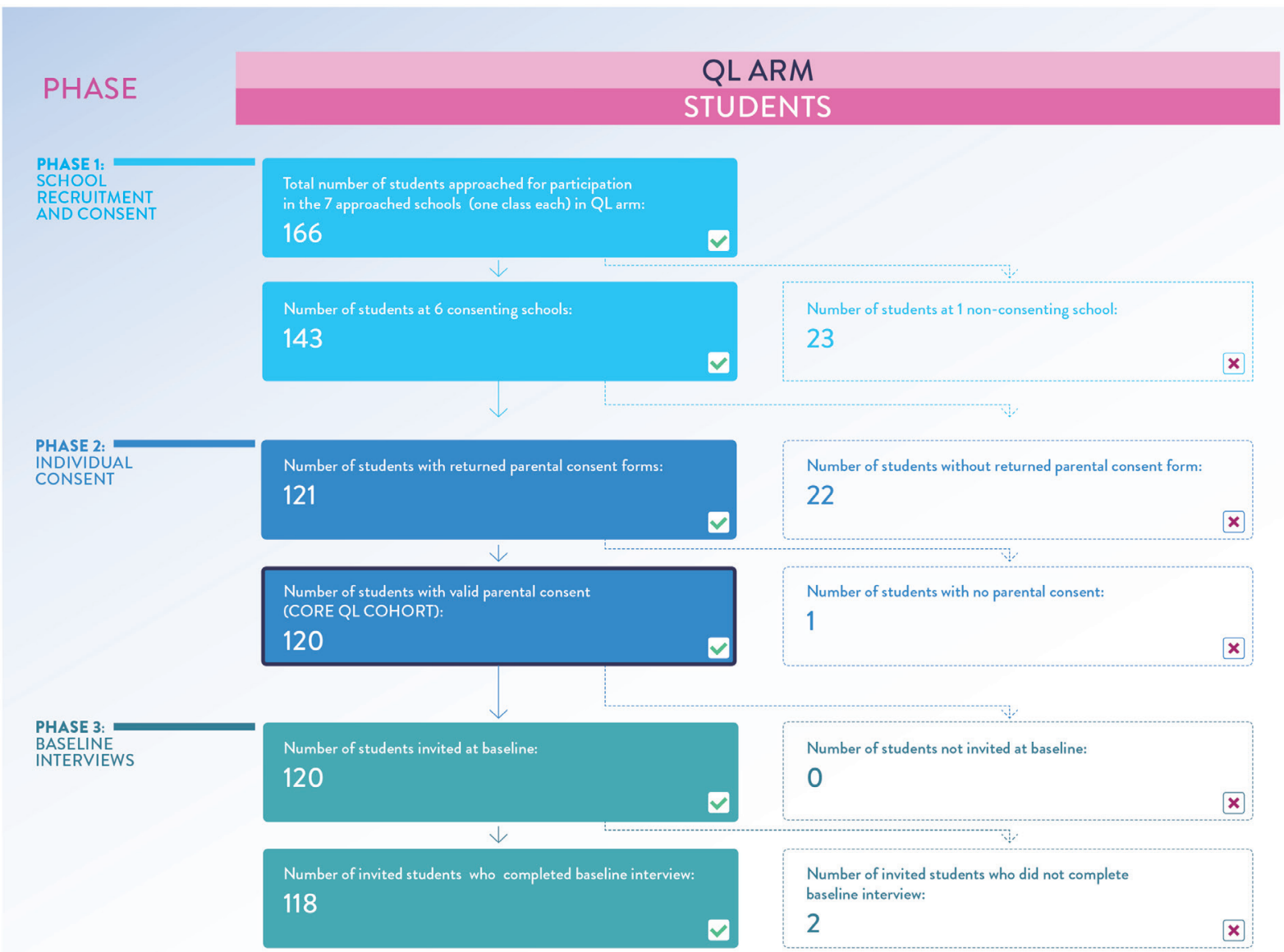

Figure 4 Flow chart for the recruitment of the qualitative arm (QL) of the MyLife study.

\section{QT consent}

Parents were asked to consent to their child(ren)'s participation in all of the planned five annual rounds of the QT arm. This involved completing a $30 \mathrm{~min}$ questionnaire during a school hour while they were in middle school, and in a private setting after they graduated middle school. The consent also involved permission for the individual-level linkages of the obtained QT data to the data available in the national health (eg, information on primary and specialised health utilisation) and other registries (eg, information on education, welfare, and unemployment) pending necessary ethical and research approvals. In addition, parents were asked to consent to be invited to complete a brief electronic questionnaire of their own and, if so, to future registry linkages as well.

In the beginning of the 2017 fall semester, 4195 valid QT consent forms were returned wherein the explicit parental consent was obtained for 3512 children. A total of 3035 parents also gave consent for their own study participation. The individual consent rate varied widely across schools, ranging from $23.2 \%$ to $96.1 \%$. The inclusion flow for the QT arm is shown in figure 3, including the baseline assessment.
QL consent

A subgroup of 143 students from six schools (one eighth grade class/cohort per school) were approached in 2015 following the main school recruitment procedures. Similar to the QT procedures above, parents were asked to consent to their child's participation in the QL arm involving both individual and group interviews over time. Parental consent for the QL arm participation was obtained for 120 students (see figure 4).

\section{Patient and public involvement}

This study involves no patients. School principals and contact persons also provided feedback after the baseline data collection, which aided the fine-tuning of follow-up procedures. Study progress and selected aggregate results are shared with those directly involved (eg, schools, parents, adolescents) through semi-annual newsletters, and with general public via the project website at the Norwegian Institute of Public Health (https://www.fhi. no/studier/mittliv/).

\section{Data collection}

Pilot study

A small scale mixed-methods pilot study including four middle schools and one high school $(n=851)$ was 
conducted in 2014 to test recruitment strategies, modes of data collection and questionnaire/interview content for both study arms. The QT pilot involved five cohorts: eighth, ninth and tenth grade middle school students, and first and second year high school students. The results informed several key aspects of the main study. For example, high school cohorts were eliminated from baseline inclusion as scientifically and logistically non-efficient; questionnaires were simplified to be appropriate for younger adolescents and several sensitive items were removed (eg, suicidality module); reimbursement strategies were fine-tuned to include gift cards of meaningful value for individual participation in combination with random lottery drawings of highly prized items such as iPhones; and National Identity Numbers were selected as the primary means of participant identification and data linkages.

The QL pilot included two eighth grade classes $(n=36)$, and it also tested recruitment strategies, interview topics, and assessment techniques. The pilot informed the main study decisions concerning the timing and balance of individual and group interviews, group sizes and composition, etc. Importantly, one of the pilot classes $(n=13)$ continued participation, and is included in the core QL sample even though its baseline was completed 1 year ahead of the main study schedule. Specifically, interviews with these participants continue to inform the decisions on how to proceed with the rest of the core sample, including the selection of emerging topics for both QT surveys and QL interviews.

The pilot characteristics and selected results are described elsewhere. ${ }^{45495558}$

\section{QT data collection}

\section{QT baseline}

During the first wave of QT data collection, the entire cohort of 3512 students was invited to complete a $30 \mathrm{~min}$ electronically-administered questionnaire during a regular school hour. All but one of the 33 invited schools managed to organise data collection. Due to this one withdrawn school ( $n_{\text {enrolled }}=65$ ), a total of 3447 instead of the eligible 3512 students were invited (see figure 3 also). Teachers supervised data collection during class time, by reading aloud a standardised MyLife study guide which described again the purpose of the study, assent and confidentiality; provided practical information about accessing the online questionnaire; and reminded the students that they are free to decline participation or withdraw from the study altogether (including the right to have information collected about them deleted). Students were instructed to type in a Uniform Resource Locator (URL) in their web browsers, which gave them access to the questionnaire. Those without parental consent were instructed to do schoolwork for the duration of the class. A contact person at each school received 1000 Norwegian Kroner (about 100 Euros) as compensation for assisting with data collection, while each participating class received the same amount into their class' savings account regardless of the individual response rates.

The questionnaire assessed a wide range of topics and characteristics, with a particular focus on family background, leisure time activities, personal characteristics, and substance use. An overview of the baseline master questionnaire is presented in table 1 . The questionnaire contained sufficient contact details to allow student identification in the Norwegian Population Registry and facilitate linkages to the unique National Identity Numbers. After removing the cases with insufficient contact information and duplicates, a total of 2975 valid responses remained, yielding a response rate of $85 \%$. For eighth, ninth and tenth graders, the response rate was $88 \%, 81 \%$ and $85 \%$, respectively.

\section{QT first annual follow-up}

By the 2018 all semester, students who were in $10^{\text {th }}$ grade at study entry in 2017 had graduated middle school and most had entered high school. For those who were still in middle school $(n=2515)$, the data collection procedure remained the same as at baseline, with the exception that the teachers handed them a secure note with a unique preassigned PIN-code to enter in the questionnaire.

Those who had graduated middle school $(n=997)$ could no longer be assessed in structured school settings, and were approached individually. First, a newsletter with updated information about the MyLife study and about the upcoming round of data collection was mailed to their home addresses. Shortly after, an email invitation with a link to the questionnaire was sent to each student, including the information that their time and effort would be reimbursed with a 200 Norwegian Kroner gift card (about 20 Euros). Three reminders were sent to non-responders via email and SMS during the fall of 2018.

The first QT follow-up was completed by 2857 adolescents (ie, $81 \%$ of the cohort). For the ninth and tenth graders who again responded in school setting, the response rate was $85.4 \%$ and $84.4 \%$, respectively. For those who were approached individually, the response rate was $72.3 \%$. Out of the entire QT cohort $(n=3512)$, $5.5 \%$ had not participated in 2017 or in $2018(\mathrm{n}=194)$; $13.1 \%(\mathrm{n}=461)$ had participated only in 2017 and $9.8 \%$ $(\mathrm{n}=343)$ had participated only in 2018. A total of $71.6 \%$ ( $\mathrm{n}=2514)$ had participated both in the 2017 baseline and in the 2018 follow-up.

\section{QL data collection \\ QL baseline}

The first wave of QL data collection was initiated in 2015 with five newly enrolled classes of eighth graders. In addition, one class completed baseline interviews in 2014, as part of the pilot study (see figure 4). This translated into a total of six eighth grade classes from six schools $(n=118)$. All baseline interviews were group interviews of approximately 45 min duration; a total of 26 interview sessions were completed. QL baseline reimbursements for both 
Table 1 Brief overview of topics encompassed in the MyLife quantitative arm adolescent baseline questionnaire (2017)

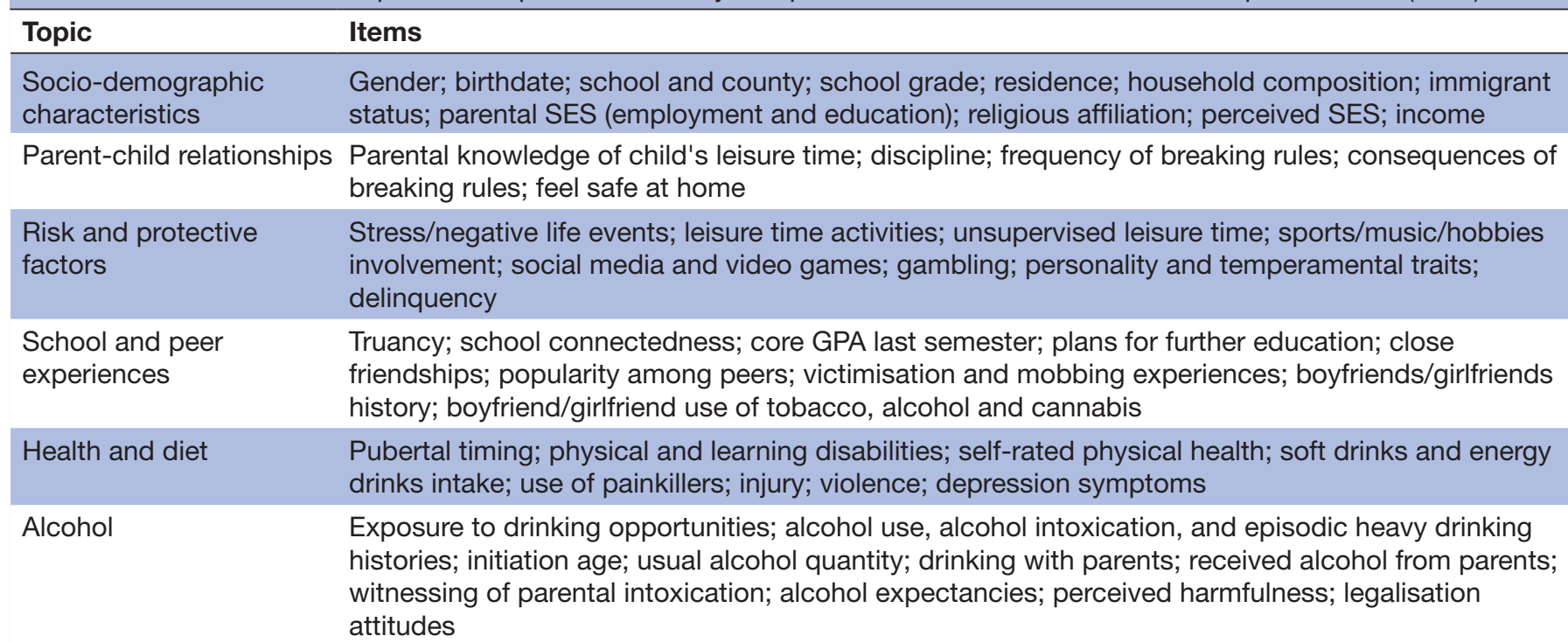

Tobacco
place of purchase; beliefs about cigarette smoking; witnessing of parental cigarette and snus use;
perceived harmfulness; legalisation attitudes

Cannabis $\quad$ Exposure to cannabis use opportunities; cannabis use history; initiation age; willingness to try cannabis; perceived cannabis availability; cannabis use expectancies; perceived harmfulness; legalisation attitudes

Other substances Synthetic cannabinoids; ecstasy; amphetamines; cocaine; prescription medications

GPA, grade point average; SES, socioeconomic status.

the student participants and teacher assistants were identical to the QT procedures.

\section{QL follow-ups}

The first QL follow-up (Time 2; 2017 Fall semester) of the five core classes was conducted when students were in the $10^{\text {th }}$ grade (ie, 2017 Fall semester). These were group interviews similar to the baseline. Interviews were conducted in smaller groups of four to six students each; a total of 24 group interviews were conducted with 85 students. The first follow-up with the one pilot class was conducted during the 2015 Spring semester, when those students were in the second semester of the eighth grade $(\mathrm{n}=13)$.

The second follow-up for all six participating classes was conducted during the second semester of their respective $10^{\text {th }}$ grade (Time 3; 2018 Spring semester for the five core classes; 2017 Spring semester for one pilot class). These were individual interviews; a total of 95 interviews of approximately $40 \mathrm{~min}$ duration were conducted during regular school hours. Participants were reimbursed with a 300 Norwegian Kroner (approximately 30 Euros) gift card for their time and effort invested into individual interviews.

\section{Additional data collection and data sources} QT parental baseline

A total of 3035 parents had consented to their own participation in the study. Of these, 2918 provided sufficient contact information and were thus invited in late 2017 to take part in the MyLife study with an email containing a questionnaire link. Two reminder emails were sent to non-responders, and by the end of the data collection in December 2017, 1899 parents had completed the questionnaire. A total of 276 parents had two children, and two parents had three children who participated in the MyLife QT arm. Therefore, parents of a total of 2041 QT children completed parental questionnaires. Parents were not reimbursed.

The content of the parental questionnaire is summarised in table 2. This $15 \mathrm{~min}$ questionnaire included items concerning the demographic, health, and well-being of the responding parent (the mother in $79 \%$ of cases) and the other parent in the household (if any), as well as the items concerning the MyLife participating child(ren).

\section{QT school administrative survey}

At QT baseline, school administrators from all recruited schools were invited to complete a brief online survey. Out of the 34 schools, 21 responded, providing both the feedback regarding their MyLife participation experiences and additional information about their school characteristics; for example, the type of prevention programme implemented (if any), school rules and disciplinary measures, distance to alcohol outlets, etc. 
Table 2 Brief overview of areas encompassed in the MyLife quantitative arm parental baseline questionnaire (2017)

\begin{tabular}{|c|c|}
\hline Topic & Items \\
\hline \multicolumn{2}{|c|}{ Information reported about self } \\
\hline $\begin{array}{l}\text { Socio- } \\
\text { demographic } \\
\text { characteristics }\end{array}$ & $\begin{array}{l}\text { Gender; date of birth; household } \\
\text { composition; own and partner's } \\
\text { education; own and partner's } \\
\text { employment status; household } \\
\text { income; home ownership; perceived } \\
\text { SES }\end{array}$ \\
\hline $\begin{array}{l}\text { Risk and } \\
\text { protective factors }\end{array}$ & $\begin{array}{l}\text { Physical and mental health; } \\
\text { relationship satisfaction; agreement } \\
\text { re: important decisions; agreement } \\
\text { re: child-rearing; negative life events; } \\
\text { personality traits }\end{array}$ \\
\hline Substance use & $\begin{array}{l}\text { Tobacco, snus, alcohol, and cannabis } \\
\text { use histories; drinking with child(ren) } \\
\text { present; house rules about smoking; } \\
\text { legalisation opinions; knowledge of } \\
\text { and attitudes towards child(ren)'s } \\
\text { possible substance use }\end{array}$ \\
\hline \multicolumn{2}{|c|}{ Information reported about the child } \\
\hline $\begin{array}{l}\text { Socio- } \\
\text { demographic } \\
\text { characteristics }\end{array}$ & $\begin{array}{l}\text { Gender; date of birth; twin status; } \\
\text { nature of relationship with child }\end{array}$ \\
\hline $\begin{array}{l}\text { Child } \\
\text { characteristics }\end{array}$ & $\begin{array}{l}\text { Learning and physical disabilities; } \\
\text { temperament; conduct problems }\end{array}$ \\
\hline $\begin{array}{l}\text { Parent-child } \\
\text { relationships }\end{array}$ & $\begin{array}{l}\text { Parental knowledge of child's leisure } \\
\text { time; discipline; frequency of breaking } \\
\text { rules; consequences of breaking rules }\end{array}$ \\
\hline $\begin{array}{l}\text { School and peer } \\
\text { experiences }\end{array}$ & $\begin{array}{l}\text { Interacting with other parents at } \\
\text { school; knowing other children at } \\
\text { school }\end{array}$ \\
\hline
\end{tabular}

SES, socioeconomic status.

\section{FINDINGS TO DATE}

\section{Core cohort characteristics}

A brief overview of the core cohort characteristics is shown in table 3A (for the QT cohort; $\mathrm{n}=3512$ ) and in table 3B (for the QL cohort, $\mathrm{n}=120$ ).

\section{QT preliminary results}

Descriptive statistics for selected demographic and substance use variables for adolescent QT baseline are presented in table 4, including basic distributions by gender and grade cohorts. The majority of responders were born in Norway, and had parents who lived together and were employed; many assigned high social status to their family when compared with other families in the neighbourhood.

In terms of the observed key outcomes, about 1 in 6 had consumed alcohol in their lifetime, but only 1 in 16 had consumed alcohol in the last 30 days. While $9 \%$ reported having tried snus, only about one-third of those reported using it in the last 30 days. This was also the case for cigarette smoking - most of those who had ever tried
Table 3 Brief overview of the MyLife core cohort characteristics

\begin{tabular}{lll}
\hline A: $(\mathbf{N}=3512)$ & & \\
\hline Characteristics & N & $\%$ \\
\hline Gender & & \\
Girls & 1923 & $54.8 \%$ \\
Boys & 1589 & $45.2 \%$
\end{tabular}

Cohort (middle school grade at

baseline inclusion)

\begin{tabular}{|ccc}
\hline Grade 8 & 1295 & $36.9 \%$ \\
\hline Grade 9 & 1220 & $34.7 \%$ \\
\hline Grade 10 & 997 & $28.4 \%$ \\
\hline County & & \\
\hline Buskerud (east) & 497 & $14.2 \%$ \\
\hline Møre og Romsdal (west) & 568 & $16.2 \%$ \\
\hline Rogaland (south) & 1137 & $32.4 \%$ \\
\hline Sør-Trøndelag (middle) & 949 & $27.0 \%$ \\
\hline Troms (north) & 361 & $10.3 \%$ \\
\hline School location & & \\
\hline Rural municipality & 1293 & $36.8 \%$ \\
\hline Town/city & 2219 & $63.2 \%$ \\
\hline Municipal/city district standard of living tertile & \\
\hline Low & 956 & $27.2 \%$ \\
\hline Medium & 1394 & $39.7 \%$ \\
\hline High & 1162 & $33.1 \%$ \\
\hline B: (N=120) & & \\
\hline Characteristics & $51.7 \%$ \\
\hline Gender & & $48.3 \%$ \\
\hline Girls & & \\
\hline Boys & & \\
\hline Cohort (middle school & & \\
\hline
\end{tabular}

Cohort (middle school grade at

baseline inclusion)

\begin{tabular}{|c|c|c|}
\hline Grade 8 & 120 & $100 \%$ \\
\hline (Fall semester 2014) & 13 & $11 \%$ \\
\hline (Fall semester 2015) & 107 & $89 \%$ \\
\hline \multicolumn{3}{|l|}{ Province } \\
\hline East & 34 & $25.8 \%$ \\
\hline West & 53 & $40.0 \%$ \\
\hline North & 33 & $34.1 \%$ \\
\hline \multicolumn{3}{|l|}{ School location } \\
\hline Rural municipality & 30 & $25.0 \%$ \\
\hline Town/city & 90 & $75.0 \%$ \\
\hline \multicolumn{3}{|c|}{ Municipal/city district standard of living tertile } \\
\hline Low & 43 & $35.8 \%$ \\
\hline Medium & 34 & $28.3 \%$ \\
\hline High & 43 & $35.8 \%$ \\
\hline
\end{tabular}


Table 4 MyLife core QT cohort demographic and key substance use outcomes at baseline $(N=2975)$

\begin{tabular}{|c|c|c|c|c|c|c|}
\hline & $\begin{array}{l}\text { Total } \\
(n=2975)\end{array}$ & $\begin{array}{l}\text { Girls } \\
(n=1668)\end{array}$ & $\begin{array}{l}\text { Boys } \\
(n=1307)\end{array}$ & $\begin{array}{l}\text { Grade } 8 \\
(n=1141)\end{array}$ & $\begin{array}{l}\text { Grade } 9 \\
(n=989)\end{array}$ & $\begin{array}{l}\text { Grade } 10 \\
(n=845)\end{array}$ \\
\hline \multicolumn{7}{|l|}{$\begin{array}{l}\text { Adolescent self-reported demographic } \\
\text { variables }\end{array}$} \\
\hline Age & $13.9(0.82)$ & $13.9(0.81)$ & $13.9(0.83)$ & $13.0(0.09)$ & $14.0(0.09)$ & $15.0(0.09)$ \\
\hline Born in Norway & $93.4 \%$ & $93.5 \%$ & $93.3 \%$ & $93.2 \%$ & $93.7 \%$ & $93.2 \%$ \\
\hline Parents live together & $72.1 \%$ & $71.1 \%$ & $73.4 \%$ & $73.2 \%$ & $70.5 \%$ & $72.5 \%$ \\
\hline \multicolumn{7}{|l|}{ Mother employed: } \\
\hline Yes & $90.1 \%$ & $90.6 \%$ & $89.4 \%$ & $89.4 \%$ & $90.3 \%$ & $90.7 \%$ \\
\hline No & $7.2 \%$ & $6.8 \%$ & $7.6 \%$ & $7.2 \%$ & $6.8 \%$ & $7.5 \%$ \\
\hline Unknown & $2.8 \%$ & $2.6 \%$ & $3.0 \%$ & $3.4 \%$ & $2.9 \%$ & $1.8 \%$ \\
\hline \multicolumn{7}{|l|}{ Father employed: } \\
\hline Yes & $92.5 \%$ & $92.4 \%$ & $92.5 \%$ & $92.2 \%$ & $92.4 \%$ & $92.9 \%$ \\
\hline No & $4.1 \%$ & $4.4 \%$ & $3.8 \%$ & $3.8 \%$ & $4.2 \%$ & $4.4 \%$ \\
\hline Unknown & $3.4 \%$ & $3.2 \%$ & $3.7 \%$ & $4.0 \%$ & $3.5 \%$ & $2.6 \%$ \\
\hline $\begin{array}{l}\text { Subjective social status (1=worst off, } \\
10=\text { best off) }\end{array}$ & $7.29(1.72)$ & 7.19 (1.68) & $7.41(1.76)$ & $7.3(1.7)$ & $7.2(1.7)$ & $7.3(1.7)$ \\
\hline \multicolumn{7}{|l|}{ Adolescent self-reported substance use } \\
\hline Ever consumed alcohol† & $15.7 \%$ & $15.3 \%$ & $16.3 \%$ & $9.3 \%$ & $14.2 \%$ & $25.9 \%$ \\
\hline Consumed alcohol in the last 30 days $\dagger$ & $6.1 \%$ & $6.5 \%$ & $5.6 \%$ & $2.3 \%$ & $4.4 \%$ & $13.2 \%$ \\
\hline Ever tried snusł† & $8.9 \%$ & $7.4 \%$ & $10.8 \%$ & $3.6 \%$ & $10.7 \%$ & $13.9 \%$ \\
\hline Used snus in the last 30 days $\dagger$ & $3.6 \%$ & $3.0 \%$ & $4.3 \%$ & $1.0 \%$ & $4.4 \%$ & $6.0 \%$ \\
\hline Ever tried a cigaretteł† & $8.6 \%$ & $7.11 \%$ & $10.5 \%$ & $4.0 \%$ & $9.1 \%$ & $14.1 \%$ \\
\hline Smoked a cigarette in the last 30 days $\dagger$ & $3.0 \%$ & $2.7 \%$ & $3.4 \%$ & $1.6 \%$ & $3.2 \%$ & $4.7 \%$ \\
\hline Tried cannabisł† & $1.3 \%$ & $0.7 \%$ & $2.0 \%$ & $0.2 \%$ & $1.4 \%$ & $2.5 \%$ \\
\hline Tried other illicit drug & $0.9 \%$ & $0.7 \%$ & $1.1 \%$ & $0.6 \%$ & $1.1 \%$ & $0.8 \%$ \\
\hline
\end{tabular}

${ }^{*}$ Gender $\left(X^{2}\right.$-tests) and cohort (logistic regressions) differences assessed for substance use outcomes only. †Statistically significant cohort differences at the $p<0.05$ level. $\ddagger$ Statistically significant gender differences at the $p<0.05$ level.

smoking had not smoked a cigarette in the last 30 days. Reported experimentation with cannabis and other illicit drugs was minimal.

Boys were more likely to report having tried snus, cigarettes, and cannabis, but there were no gender differences in alcohol consumption, snus use, and cigarette smoking in the last 30 days. All substance use outcomes, save for trying illicit drugs, were more prevalent in older cohorts, as expected.

Table 5 Current substance use in 2017 and 2018 for those who participated in both quantitative arm waves, $N=2514$

\begin{tabular}{lllll}
\hline Current use of: & Never & $\begin{array}{l}\text { Only in } \\
\mathbf{2 0 1 7}\end{array}$ & $\begin{array}{l}\text { Only in } \\
\mathbf{2 0 1 8}\end{array}$ & $\begin{array}{l}\text { In 2017 } \\
\text { and 2018 }\end{array}$ \\
\hline Alcohol & $66.7 \%$ & $1.6 \%$ & $23.2 \%$ & $8.5 \%$ \\
Snus & $86.7 \%$ & $2.6 \%$ & $7.2 \%$ & $3.5 \%$ \\
Cigarette & $92.7 \%$ & $1.4 \%$ & $4.8 \%$ & $1.2 \%$ \\
Cannabis & $96.1 \%$ & $0.4 \%$ & $2.8 \%$ & $0.7 \%$ \\
\hline
\end{tabular}

Current use, used at least once in the last 12 months. table 5 shows substance use outcomes in 2017 and 2018 for respondents at both time points to assess stability in use, as well as the rate of starters and quitters. By 2018, a large majority had still never consumed alcohol, or tried snus, cigarettes, or cannabis. However, almost one-fourth of the respondents started to drink alcohol during this time period. Substantially fewer initiated the use of snus $(7.2 \%)$, cigarettes $(4.8 \%)$, and cannabis $(2.8 \%)$. Proportions of those who ceased using alcohol, snus, cigarettes, and cannabis between 2017 and 2018 were minimal (all $<3 \%)$.

\section{QL preliminary results}

Pilot study interviews indicated that the participants 12 to 13 year old at that time - were highly sceptical of drinking. They believed that young people drink because they want to be cool, because they are miserable, or because of group pressure. These narratives were interpreted as reflecting a deep-rooted cultural ambivalence towards alcohol use in Norway while also suggesting that boundaries between 'adolescents' and 'adults' are 
fundamental when understanding emerging adolescent social identities, especially when it comes to drinking and drinking culture. ${ }^{58}$

Preliminary analysis of the main study interviews suggested a normalisation of non-drinking among 15 to 16 year olds, and continued ambivalence in their perceptions of alcohol use. While cigarette smoking was very uncommon, snus use was more common and socially accepted; e-cigarettes seemed to have some novelty value. Overall, substance use appeared not to be at the core of many adolescents' self-image, which often centred on school performance, sports and exercise, and ambitions and plans for the future. However, some individual trajectories highlighted vulnerability that may point towards future problem use of alcohol and/or illegal substances.

\section{STRENGTHS AND LIMITATIONS}

To the best of our knowledge, MyLife is the first longitudinal study of youth in Norway to employ quantitative developmental assessments in combination with qualitative individual and group interviewing in order to specifically examine substance use over the early lifespan. The MyLife project aims to integrate multiple sources of data, multiple informants and multiple methodologies. In that respect, the project will significantly (a) extend and augment the knowledge gained by previous studies of adolescent health and adjustment in general, and of substance use in particular, (and b) contribute to both national and international research and public health policy.

As with all studies, there are important limitations. First, the non-alignment of the QT and QL baselines resulted from administrative delays; nevertheless, the project largely retained its multidisciplinary and mixed-methods character. Next, because of the privacy and confidentiality concerns, the participating schools were not able to share parental contact info with the MyLife team. For this reason, distribution of the project information booklets and consent forms was outsourced to schools and ultimately to students themselves. Given this reliance on adolescents as liaisons, it is not known how many parents were properly informed about the MyLife study. It is possible that the more vulnerable or high-risk adolescents were less likely to share this info with their parents, thus affecting the core sample characteristics. This also suggests that the utilised consent strategy, although the only ethically feasible one in this case, might have been less than optimal.

The related limitation concerns inferences and generalisability of our results. For example, given our inclusion criteria and sampling strategies, we may have missed the onset of substance use before grade 8 and among most vulnerable adolescents. However, we can still identify those 'early starters' in the core sample. Second, we do not aim to estimate national prevalence rates or to draw related inferences. Large-scale national monitoring efforts, such as the European School Survey
Project on Alcohol and Other Drugs (ESPAD), ${ }^{71}$ are far more appropriate when population-level estimates and population-level generalisability are of primary interest. Rather, MyLife aims to draw inferences about the associations between the putative risk and protective factors and various substance use outcomes and patterns over time. In such cases, population-representative samples are not required, given appropriate control for confounding and avoidance of other biasses. ${ }^{72}$ Relatedly, a major criticism of longitudinal studies with non-representative samples is the lack of heterogeneity. ${ }^{73}$ In our case, over-sampling from different geographical and socioeconomic areas supports greater cohort heterogeneity than would have been obtained through the basic random sampling. Finally, identification of the factors associated with attrition may improve future retention rates, especially for those participants followed-up individually.

\section{Future plans}

Both study arms have planned individual adolescent follow-ups through 2021 (see figure 1). Specifically, three more QT annual rounds are scheduled for 2019, 2020, and 2021. The planned procedures and data collection strategies are identical as for the 2018, but after 2019 the entire cohort - having graduated middle school - will be contacted and assessed individually. QL follow-ups will be conducted as individual interviews, specifically during the spring of 2019 (ie, the first year of high school), and spring 2021 (ie, the final year of high school).

Norway has rich information about each of its municipalities, particularly indicators of socioeconomic status such as the unemployment rate, income disparity, and educational level. Similar information is available for schools, in addition to the information provided by school administrators. These characteristics can be linked to both aggregate-level and individual-level data and be included in the planned mixed-methods and multilevel analyses.

Further, parental consent was obtained for individual-level linkages of questionnaire data to the national-level administrative sources of data, such as health, education, and labour and social registries. ${ }^{60}$ These registries can provide additional information on putative risk exposures, as well as on the range of long-term outcomes for MyLife adolescents through continually and prospectively updated individual registry records. Registry linkages will be made for all cohort members, including parents, following the necessary administrative approvals; identifying National Identity Numbers have already been obtained from the Population Registry.

Acknowledgements We thank all participating students and their families, as well as all the schools and teachers who assisted with the MyLife data collection. The authors would like to acknowledge the important contributions to the MyLife project made by the following individuals: Camilla Stub Lundberg, Mads Toftgaard, Silje Bakken, Øystein Skjælaaen, Skule Wigenstad, Marie Svendsen Nerheim, Tilmann von Soest, and Kristina Jackson.

Contributors GSB and JBA drafted the manuscript, with substantive contributions from JS, RT and KB. JBA, EK and GSB designed and established the Quantitative 
arm of the MyLife project, while JS and RT designed and established the Qualitative arm. JBA, GSB and JS remain responsible for the scientific direction and continued management of the project as a whole, with the assistance from EK, RT and KB. All authors reviewed, critically revised, and approved the manuscript.

Funding The authors have not declared a specific grant for this research from any funding agency in the public, commercial or not-for-profit sectors.

Competing interests None declared.

Patient consent for publication Not required.

Ethics approval The MyLife project received approval by the Norwegian Data Protection Authority (DPA) (reference no.: 15/01495) after ethical evaluation by The National Committee for Research Ethics in the Social Sciences and the Humanities (reference no.: 2016/137). All participants were fully informed about the study. Additional approvals from relevant authorities will be sought on an as-needed basis for future individual-level data linkages (ie, national social and health registries) and for potential study extensions.

Provenance and peer review Not commissioned; externally peer reviewed.

Data availability statement Researchers interested in collaboration are both welcomed and invited to propose research projects by contacting the MyLife team. The MyLife dataset is administrated by the Norwegian Institute of Public Health

Open access This is an open access article distributed in accordance with the Creative Commons Attribution Non Commercial (CC BY-NC 4.0) license, which permits others to distribute, remix, adapt, build upon this work non-commercially, and license their derivative works on different terms, provided the original work is properly cited, appropriate credit is given, any changes made indicated, and the use is non-commercial. See: http://creativecommons.org/licenses/by-nc/4.0/.

\section{ORCID iD}

Jasmina Burdzovic Andreas http://orcid.org/0000-0002-6730-1321

\section{REFERENCES}

1 Aarons GA, Brown SA, Coe MT, et al. Adolescent alcohol and drug abuse and health. J Adolesc Health 1999;24:412-21.

2 U.S. Department of Health and Human Services OotSG. The Surgeon General's Call to Action To Prevent and Reduce Underage Drinking 2007.

3 Moss HB, Chen CM, Yi H-Y. Early adolescent patterns of alcohol, cigarettes, and marijuana polysubstance use and young adult substance use outcomes in a nationally representative sample. Drug Alcohol Depend 2014;136:51-62.

4 Degenhardt L, Charlson F, Ferrari A, et al. The global burden of disease attributable to alcohol and drug use in 195 countries and territories, 1990-2016: a systematic analysis for the global burden of disease study 2016. Lancet Psychiatry 2018:5:987-1012.

5 Institute for Health Metrics and Evaluation. GBD compare Seattle. Seattle, WA: IHME, University of Washington, 2018. http://vizhub. healthdata.org/gbd-compare

6 Erskine HE, Moffitt TE, Copeland WE, et al. A heavy burden on young minds: the global burden of mental and substance use disorders in children and youth. Psychol Med 2015;45:1551-63.

7 Hawkins JD, Catalano RF, Miller JY. Risk and protective factors for alcohol and other drug problems in adolescence and early adulthood: implications for substance abuse prevention. Psychol Bull 1992;112:64-105.

8 Sloboda Z, Glantz MD, Tarter RE. Revisiting the concepts of risk and protective factors for understanding the etiology and development of substance use and substance use disorders: implications for prevention. Subst Use Misuse 2012;47:944-62.

9 Stone AL, Becker LG, Huber AM, et al. Review of risk and protective factors of substance use and problem use in emerging adulthood. Addict Behav 2012;37:747-75.

10 Chassin L, Pitts SC, Prost J. Binge drinking trajectories from adolescence to emerging adulthood in a high-risk sample: predictors and substance abuse outcomes. J Consult Clin Psychol 2002;70:67-78.

11 Jackson KM, Sher KJ, Schulenberg JE. Conjoint developmental trajectories of young adult substance use. Alcoholism Clin Exp Res 2008;32:723-37

12 Orlando M, Tucker JS, Ellickson PL, et al. Concurrent use of alcohol and cigarettes from adolescence to young adulthood: an examination of developmental trajectories and outcomes. Subst Use Misuse 2005;40:1051-69.
13 Patton GC, Coffey C, Lynskey MT, et al. Trajectories of adolescent alcohol and cannabis use into young adulthood. Addiction 2007;102:607-15.

14 Guo J, Collins LM, Hill KG, et al. Developmental pathways to alcohol abuse and dependence in young adulthood. J Stud Alcohol 2000;61:799-808.

15 Chen P, Jacobson KC. Developmental trajectories of substance use from early adolescence to young adulthood: gender and racial/ethnic differences. J Adolesc Health 2012;50:154-63.

16 Englund MM, Egeland B, Oliva EM, et al. Childhood and adolescent predictors of heavy drinking and alcohol use disorders in early adulthood: a longitudinal developmental analysis. Addiction 2008;103:23-35.

17 Clark DB. The natural history of adolescent alcohol use disorders. Addiction 2004:99:5-22.

18 Settles RE, Smith GT. Toward a developmentally centered approach to adolescent alcohol and substance use treatment. Curr Drug Abuse Rev 2015;8:134-51.

19 Glantz MD, Leshner Al. Drug abuse and developmental psychopathology. Dev Psychopathol 2000;12:795-814.

20 Chassin L, Sher KJ, Hussong A, et al. The developmental psychopathology of alcohol use and alcohol disorders: research achievements and future directions. Dev Psychopathol 2013;25:1567-84.

21 Thomas YF. The social epidemiology of drug abuse. Am J Prev Med 2007;32:S141-6.

22 Ennett ST, Foshee VA, Bauman KE, et al. The social ecology of adolescent alcohol misuse. Child Dev 2008;79:1777-91.

23 Galea S, Nandi A, Vlahov D. The social epidemiology of substance use. Epidemiol Rev 2004;26:36-52.

24 Bronfenbrenner U. Toward an experimental ecology of human development. Am Psychol 1977;32:513-31.

25 Costello EJ, Angold AC. Developmental Epidemiology. In: Sameroff AJ, Lewis M, Miller SM, eds. Handbook of developmental psychopathology. Boston, MA: Springer US, 2000: 57-73.

26 Hser Y-I, Longshore D, Anglin MD. The life course perspective on drug use: a conceptual framework for understanding drug use trajectories. Eval Rev 2007;31:515-47.

27 Boyce WT, Frank E, Jensen PS, et al. Social context in developmental psychopathology: recommendations for future research from the MacArthur network on psychopathology and development. The MacArthur Foundation research network on psychopathology and development. Dev Psychopathol 1998;10:143-64.

28 Castellanos-Ryan N, O'Leary-Barrett M, Conrod PJ. Substance-Use in childhood and adolescence: a brief overview of developmental processes and their clinical implications. J Can Acad Child Adolesc Psychiatry 2013;22:41-6.

29 Palmer RHC, Young SE, Hopfer CJ, et al. Developmental epidemiology of drug use and abuse in adolescence and young adulthood: evidence of generalized risk. Drug Alcohol Depend 2009;102:78-87

30 Swift W, Coffey C, Carlin JB, et al. Adolescent cannabis users at 24 years: trajectories to regular Weekly use and dependence in young adulthood. Addiction 2008;103:1361-70.

31 Yoshikawa $\mathrm{H}$, Weisner TS, Kalil A, et al. Mixing qualitative and quantitative research in developmental science: uses and methodological choices. Dev Psychol 2008;44:344-54.

32 Sullivan ML. Integrating qualitative and quantitative methods in the study of developmental psychopathology in context. Dev Psychopathol 1998;10:377-93.

33 Creswell JW KA, Plano Clark VL, Smith KC. Best practices for mixed methods research in the health sciences. National Institutes of Health, 2011.

34 Teddlie C, Tashakkori A. Foundations of mixed methods research: integrating quantitative and qualitative approaches in the social and behavioral sciences. SAGE Publications, 2009.

35 Nathan S, Rawstorne P, Hayen A, et al. Examining the pathways for young people with drug and alcohol dependence: a mixed-method design to examine the role of a treatment programme. BMJ Open 2016;6:e010824.

36 Østergaard J. Learning to become an alcohol user: adolescents taking risks and parents living with uncertainty. Addict Res Theory 2009;17:30-53.

37 Folkehelseinstituttet. Ti store folkehelseutfordringer I Norge. HVA sier analyse AV sykdomsbyrde? Oslo: Folkehelseinstituttet, 2018.

38 Erevik EK, Pallesen S, $\varnothing \mathrm{V}$, et al. Alcohol use among Norwegian students:Demographics, personality and psychological health correlates of drinking patterns. Nordic Studies on Alcohol and Drugs 2017;34:415-29. 
39 Burdzovic Andreas J, Pape $\mathrm{H}$. Who receives cannabis use offers: a general population study of adolescents. Drug Alcohol Depend 2015;156:150-6.

40 Pape H, Norström T, Rossow I. Adolescent drinking-a touch of social class? Addiction 2017;112:792-800.

41 Burdzovic Andreas J, Pape H, Bretteville-Jensen AL. Who are the adolescents saying "No" to cannabis offers. Drug Alcohol Depend 2016;163:64-70.

42 Burdzovic Andreas J, Bretteville-Jensen AL. Ready, willing, and able: the role of cannabis use opportunities in understanding adolescent cannabis use. Addiction 2017;112:1973-82.

43 Pape H, Bye EK. Drinking with parents: different measures, different associations with underage heavy drinking? Nordic Studies on Alcohol and Drugs 2017;34:445-55.

44 Pape H, Rossow I, Storvoll EE. Under double influence: assessment of simultaneous alcohol and cannabis use in general youth populations. Drug and alcohol dependence 2009;101:69-73.

45 Sandberg S, $\varnothing$ S. "Shoes on your hands": perceptions of alcohol among young adolescents in Norway. Drugs: Education, Prevention and Policy 2018;25:449-56.

46 Strandheim A, Holmen TL, Coombes L, et al. Alcohol intoxication and mental health among adolescents--a population review of 8983 young people, 13-19 years in North-Trøndelag, Norway: the YoungHUNT Study. Child Adolesc Psychiatry Ment Health 2009;3:18.

47 Brunborg GS, Norström T, Storvoll EE. Latent developmental trajectories of episodic heavy drinking from adolescence to early adulthood: predictors of trajectory groups and alcohol problems in early adulthood as outcome. Drug Alcohol Rev 2018;37:389-95.

48 Enstad F, Pedersen W, Nilsen W, et al. Predicting early onset of intoxication versus drinking-A population-based prospective study of Norwegian adolescents. Addict Behav Rep 2017;6:1-7.

49 Brunborg GS, Andreas JB, Kvaavik E. Social media use and episodic heavy drinking among adolescents. Psychol Rep 2017;120:475-90.

50 Brunborg GS, Hanss D, Mentzoni RA, et al. Core and peripheral criteria of video game addiction in the game addiction scale for adolescents. Cyberpsychol Behav Soc Netw 2015;18:280-5.

51 Mentzoni RA, Brunborg GS, Molde $\mathrm{H}$, et al. Problematic video game use: estimated prevalence and associations with mental and physical health. Cyberpsychol Behav Soc Netw 2011;14:591-6.

52 Brunborg GS, Burdzovic Andreas J. Increase in time spent on social media is associated with modest increase in depression, conduct problems, and episodic heavy drinking. J Adolesc 2019;74:201-9.

53 Hines LA, Morley KI, Strang J, et al. Onset of opportunity to use cannabis and progression from opportunity to dependence: are influences consistent across transitions? Drug Alcohol Depend 2016;160:57-64

54 Abebe DS, Hafstad GS, Brunborg GS, et al. Binge drinking, cannabis and tobacco use among ethnic Norwegian and ethnic minority adolescents in Oslo, Norway. J Immigr Minor Health 2015;17:992-1001.

55 Burdzovic Andreas J, Brunborg GS. Depressive symptomatology among Norwegian adolescent boys and girls: the patient health Questionnaire-9 (PHQ-9) psychometric properties and correlates. Front Psychol 2017;8:887.
56 Pape H, Rossow I, Brunborg GS. Adolescents drink less: how, who and why? A review of the recent research literature. Drug Alcohol Rev 2018;37 Suppl 1:S98-114.

57 Burdzovic Andreas J. Perceived harmfulness of various alcohol- and cannabis use modes: secular trends, differences, and associations with actual substance use behaviors among Norwegian adolescents, 2007-2015. Drug Alcohol Depend 2019;197:280-7.

58 Bakken SA, Sandøy TA, Sandberg S. Social identity and alcohol in young adolescence: the perceived difference between youthful and adult drinking. J Youth Stud 2017;20:1380-95.

59 Scheffels J, Moan IS, Storvoll E. Everything in moderation? a mixed methods study on perceptions of parents' drinking in the presence of children. Nordic Studies on Alcohol and Drugs 2016;33:551-66.

60 Research Council of Norway. Health registries for research (HRR): facilitating the use and security of Norwegian health registries in research, 2019. Available: https://hrr.w.uib.no/

61 Galbraith S, Bowden J, Mander A. Accelerated longitudinal designs: an overview of modelling, power, costs and handling missing data. Stat Methods Med Res 2017;26:374-98.

62 Duncan SC, Duncan TE, Hops H. Analysis of longitudinal data within accelerated longitudinal designs. Psychol Methods 1996;1:236-48.

63 Willett JB, Singer JD, Martin NC. The design and analysis of longitudinal studies of development and psychopathology in context: statistical models and methodological recommendations. Dev Psychopathol 1998;10:395-426.

64 Collins LM. Analysis of longitudinal data: the integration of theoretical model, temporal design, and statistical model. Annu Rev Psychol 2006;57:505-28.

65 Raudenbush SW, CHAN W-S, W-s C. Growth curve analysis in accelerated longitudinal designs. J Res Crime Delinq 1992;29:387-411.

66 Nagin DS, Jones BL, Passos VL, et al. Group-Based multi-trajectory modeling. Stat Methods Med Res 2018;27:2015-23.

67 Li F, Duncan TE, Hops H. Examining developmental trajectories in adolescent alcohol use using piecewise growth mixture modeling analysis. J Stud Alcohol 2001;62:199-210.

68 Grossoehme D, Lipstein E. Analyzing longitudinal qualitative data: the application of trajectory and recurrent cross-sectional approaches. BMC Res Notes 2016;9:136.

69 The Human Development Report 2010 Team. Human development report 2010, 20th anniversary edition: the real wealth of nations, pathways to human development. New York, NY: United Nations Development Programme, 2010.

70 Rogstad L. Indeks for levekårsproblemer. Samfunnsspeilet 2002;16:106-8.

71 Kraus L, Nociar A. ESPAD report 2015: results from the European school survey project on alcohol and other drugs: European monitoring centre for drugs and drug addiction 2016.

72 Rothman KJ, Gallacher JEJ, Hatch EE. Why representativeness should be avoided. Int J Epidemiol 2013;42:1012-4.

73 Richiardi L, Pizzi C, Pearce N. Commentary: representativeness is usually not necessary and often should be avoided. Int $\mathrm{J}$ Epidemiol 2013;42:1018-22. 